\title{
Validation of Neural Network Predictions for the Outcome of Refractive Surgery for Myopia
}

\author{
Miltos Balidis ${ }^{1}$, Ioanna Papadopoulou ${ }^{2}$, Dimitris Malandris ${ }^{2}$, Zachos Zachariadis ${ }^{1}$, Dimitrios Sakellaris ${ }^{1}$, Solon Asteriadis ${ }^{1}$, \\ Marios Poulos ${ }^{3}$, Zisis Gatzioufas ${ }^{4}$, George Anogeianakis ${ }^{1,5}$ \\ ${ }^{1}$ Ophthalmica Institute of Ophthalmology and Microsurgery, Thessaloniki, Greece. \\ ${ }^{2}$ Faculty of Medicine, Aristotle University of Thessaloniki, Thessaloniki, Greece. \\ ${ }^{3}$ Laboratory of Information Technologies Faculty of Information Science and Informatics, Ionian University, Corfu, Greece. \\ ${ }^{4}$ Department of Ophthalmology, Cornea, Cataract and Refractive Surgery, University Hospital Basel, Basel, Switzerland. \\ ${ }^{5}$ Association for Training in Biomedical Technology, Thessaloniki, Greece.
}

\begin{abstract}
Background: Refractive surgery (RS) for myopia has made a very big progress regarding its safety and predictability of the outcome. Still, a small percentage of operations require retreatment. Therefore, both legally and ethically, patients should be informed that sometimes a corrective RS may be required. We addressed this issue using Neural Networks (NN) in RS for myopia. This was a recently developed validation study of a NN.

Methods: We anonymously searched the Ophthalmica Institute of Ophthalmology and Microsurgery database for patients who underwent RS with PRK, LASEK, Epi-LASIK or LASIK between 2010 and 2018. We used a total of 13 factors related to RS. Data was divided into four sets of successful RS outcomes used for training the NN, successful RS outcomes used for testing the NN performance, RS outcomes that required retreatment used for training the NN and RS outcomes that required retreatment used for testing the NN performance. We created eight independent Learning Vector Quantization (LVQ) networks, each one responding to a specific query with 0 (for the retreat class) or 1 (for the correct class). The results of the 8 LVQs were then averaged so we could obtain a best estimate of the NN performance. Finally, a voting procedure was used to reach to a conclusion.

Results: There was a statistically significant agreement (Cohen's Kappa $=0.7658$ ) between the predicted and the actual results regarding the need for retreatment. Our predictions had good sensitivity (0.8836) and specificity (0.9186). Conclusions: We validated our previously published results and confirmed our expectations for the NN we developed. Our results allow us to be optimistic about the future of NNs in predicting the outcome and, eventually, in planning RS.
\end{abstract}

\section{KEY WORDS}

Neural Network; Refractive Surgical Procedure; Myopia; PRK; LASEK; Epi-LASIK; LASIK; Computer Neural Network.

Copyright (c) 2020, Author(s). This is an open-access article distributed under the terms of the Creative Commons Attribution-Non Commercial 4.0 International License (http://creativecommons.org/licenses/by-nc/4.o/) which permits copy and redistribute the material just in noncommercial usages, provided the original work is properly cited.

Correspondence to: Professor George Anogeianakis MD, PhD; Ophthalmica Institute of Ophthalmology and Microsurgery, Thessaloniki, Greece. E-mail: anogian@hotmail.com

How to cite this article: Balidis M, Papadopoulou I, Malandris D, Zachariadis Z, Sakellaris D, Asteriadis S, Poulos M, Gatzioufas Z, Anogeianakis G, Validation of Neural Network Predictions for the Outcome of Refractive Surgery for Myopia. Med Hypothesis Discov Innov Ophthalmol. 2020; 9(3): 172-178.

\section{INTRODUCTION}

Artificial Intelligence (Al) and Neural Networks (NN) are considered as powerful assistants when sensing, learning, reasoning, and making decisions are required, especially in tasks where "learning" of different tasks requires the processing of large amounts of data [1, 2]. 
In ophthalmology, for a number of retinal diseases, Deep Learning (DL) algorithms, already make referral recommendations processing 3-dimensional optical coherence tomography (OCT) data [3]. The DL recommendations exhibit equal or better validity with experts' ideas. DL algorithms, for instance, scan images for signs of diabetic retinopathy [4], have reduced the burden of interpretation of an ever-growing volume and complexity of diagnostic images. In another implementation in East and Southeast Asia, Al predicts the progression of myopia and detects high myopia in young adults, up to 8 years in advance of its onset [5]. Last but not least, a statistical classifier algorithm maximizes the use of "big data" [6] to predict refractive surgery (RS) outcome.

RS already fulfills strict criteria for safety, efficacy and cost-effectiveness [7, 8]. However, regarding the predictability of refractive outcome a small, albeit not negligible percentage of patients require a corrective retreatment [9-11]. Not only considering medical ethics, in some countries, the law also requires that patients should be well informed of any possibility that corrective RS may be required. We focused on myopia because, in our experience, myopia correction is the most broadly used refractive correction and, by far, the most popular among young adults. In previous work [12], we developed a NN to predict the possibility of retreatment in RS performed to correct myopia. In that paper, we developed the mathematical and computational tools to tackle the problem and we isolated the necessary clinical parameters from a sample of more than 4500 RS myopia procedures to train and test the $\mathrm{NN}$ that we used to detect high-risk patients. The only criterion for the selection was that we had a complete set of data, for each and every patient we selected. on the parameters that we used for NN training and testing.

The present paper was dictated by two basic considerations. First because "Replicating studies are important" [13], and there is a need "to establish nuanced solutions to improve transparency, accountability, and reporting of research]" [13] and, in addition, "Concern over the reliability of published biomedical results grows unabated" [14]. Second, by establishing the repeatability of our method and disseminating verified results, we can facilitate widely testing of our NN (which is available for anyone that requests it) in a clinical setting.

\section{METHODS}

This study adhered to the same principles as our original study [12]. We randomly selected patients who underwent RS with PRK, LASEK, Epi-LASIK and LASIK by the Ophthalmica surgeons from January 2010 to September
2018. We investigated a total of 13 RS related factors as described in our original study [12].

In our experience, success of NN training strongly depends on the way the parameters used are normalized, because the NN training is extremely sensitive to data $[12,15,16]$ of the type of the 13 parameters we use. Although, theoretically, it is not necessary to normalize independent (i.e., numerical) data, it turns out that normalization of numerical data values often makes NN training more efficient, and it makes the NN a better predictor [17].

The training and application of used DL network is based on the so called "back propagation algorithm" which involves a two-stage procedure; a forward propagation stage (recognition) and a back-propagation stage. The computations performed either during training or application of the NN depends on the multiplication of real-valued weights by a real-valued activation function (forward propagation) or by the gradient mentioned above (back-propagation) [18]. This approach to normalization, eliminates most multiplications by forcing the weights to be binary [18]. To achieve this, we chose to represent each integer by a 12-bit serial code. 12-bit encoding was used because of the size and the negativity of the integers used. Thus, following this preprocessing stage, a vector of all the data values for each patient was encoded in a $1 \times\left(13^{*} 12\right)=1 \times 156$ bit size binary vector. To classify data, i.e., to determine which myopia RS needs a retreatment, we used a Learning Vector Quantizer (LVQ) $\mathrm{NN}$, which is based on a self-organizing network approach whereby the training vectors recursively "tune" those hidden NN units which correspond to different categories of the inputs.

A trained network categorizes an input vector as member of a class which is represented by the nearest hidden unit [19]. LVQ networks perform very well in pattern classification [20]. The reason we chose an LVQ NN was that LVQ NNs are characterized by non-linear classification properties [17]. LVQ NNs comprise two layers; a first layer maps input vectors into clusters determined during the learning phase and a second layer which consolidates these clusters into classes characterized by the target data. The first step in LQV NN design deals with setting the parameters of the two NN layers. The data vectors that will be used as input are divided into a training and a test group. During learning, a "codebook Initialization" is performed first. The codebook vector is a table that represents the average of all the vectors that belong to a cluster. Our NN then compares the number of codebook vectors that correspond to each target group with the number of occurrences for that group. Following that, these vectors are adjusted to the 
center of the input range. In this way two training groups, a retreat and a non-retreat group are created. The training itself of an LVQ is a two-step procedure. During the first step, the initial positions of the codebook vectors are determined in the $r$-dimensional space, for a given number of classes using standard clustering algorithms. One such algorithm is the K-Means that partitions observations into $\mathrm{k}$ clusters so that the pairwise squared deviations of points within the same cluster are minimized. Another similar algorithm is the Linde-BuzoGray algorithm (LBG algorithm). During the second step, the codebook vectors are iteratively updated in order in an attempt to minimize the total classification error of the training set. The codebook vectors are "marshaled" in the direction of data vectors of the same class, while being distanced from the data vectors of different classes [21, 22]. Following codebook Initialization, a Winner decision is taken. The Euclidean distance is calculated between the training data vector and the codebook vector. Thus, whenever a member of the training set feature vector, is incorrectly classified, both involved correct and incorrect codebook vectors are updated. The LVQ network is selforganizing since it is determined by the weights of the competition layer and it uses the training input vectors, to reflect distribution of the sample data patterns in the output layer. The LVQ pattern recognition algorithm is resultant from the Kohonen competitive algorithm [17, 21]. The architecture of the LVQ network is depicted in Figure 1.

During testing, the network reacts with an unknown vector $P$ (see figure 1). The decision depends on the error difference between the output y vector and the reconstructed vector and it has two values; 0 for the correct class or 1 for the retreat class. It is designated as ynet.

To improve the range of the values for both sensitivity and specificity of the individual NN predictions we resorted to a Voting Procedure among a number of NN to which we posed the same question.

In effect, the Winner decision of each NN queried is followed by a voting procedure among the NNs. This is akin to an information aggregation procedure and joint decision-making by more than one NNs. In our proposed system, the new query vector is tested against each of the $\mathrm{K}$ two-way classifiers. The result is the determination, by each NN, of the class it belongs to. The correct class indicates (with a high probability) that the RS myopia will be successful; in contrast, the retreat class reflects a particularly low probability for successful RS outcome. This procedure results in $\mathrm{K}$ scores from the $\mathrm{K}$ classifiers. Obtaining an "unambiguous" (i.e., highly probable) prediction for a given testing vector $[23,24]$ is achieved by reducing or eliminating false positives. In our case, all votes from the eight classifiers we use, are tallied and the class that receives the most votes becomes the final prediction. At this second step of the decision stage, therefore, false positives are in fact eliminated. In eliminating the false positives, the decision boundary is drawn between two "true" classes of training vectors, instead of one "true" class and its complementary "others" class, which is enormously complex. These classes are determined according to the test input and the decision threshold so that:

If more than $4 \mathrm{NN}$ "vote" "correct, (i.e., 1)" for a nonretreat test vector than the result is "correct identification".

If less than 4 NN "vote" "retreat, (i.e., 0)" for a non-retreat test vector than the result is "not correct identification".

If more than 4 NN "vote" "correct, (i.e., 1)" for a retreat test vector than the result is "correct identification".

If less than 4 NN "vote" "retreat, (i.e., 0)" for a retreat test vector than the result is "not correct identification".

In this way, false positives are eliminated accurately. Also, for non-correct identification (or the cases that indeed require retreat) we set stricter criteria for securing correct identification. Thus, we adopted a larger range of votes (i.e., 0, 1, 2, 3 and 4) than the case for the correct identification to increase the specificity. The complete mathematical reasoning and development of our NN has been previously described [12].

From the January 1, 2010 to September13, 2018, a total of 5210 RS operations were performed by fifteen ophthalmic surgeons in Ophthalmica. Of these, 5117 were original treatments and 93 corrective retreatments. Overall retreatment/treatment ratio was 0.01785 , lower than the reported rates in the literature [10, 25-35].

The previously calculated correlation of the experience of the individual surgeons with their RS outcomes was ( $r$ for number of treatments vs \% of retreatments) very close to zero $(-0.06352)$, which indicates the independence of the operating surgeon experience from the outcome.

Therefore, surgeon identity was not included in the 13 factors which are related to RS and used in the construction of the binary vector of 156-bit size, which encoded the parameters for each patient, as mentioned above.

Of the above set of operations, following and matching exactly the protocol we used in our previous study, we randomly isolated from our cohort 2378 non-retreat and 70 retreat patients from those 5210 treatments who were followed-up for over a year and complete data was available for the RS parameters that we used. 
The 2378 non-retreat and 70 retreat vectors were constructed according to the Preprocessing Stage. The rest of the NN operation, strictly followed the description for Data Classification Stage and Decision Making. As mentioned, for the voting procedure, the above procedure was repeated, for every attempt to predict the RS outcome, eight times randomly, whereby each procedure represented a different training and testing NN.

\section{RESULTS}

To prove that the used neural classification test predicts the outcome of RS with a reasonable accuracy, we had to determine the validity of the prediction. To measure validity, one must use the values of sensitivity and specificity. For this a conventional two- by-two $(2 \times 2)$ table (Table 1) was used. Table 1 compares the outcome of RS that required no retreatment versus those who required retreatment. This comparison was made for each run of the NN algorithm before the voting stage is implemented.

To understand whether a substantial agreement exists between the outcome of neural classification ("observed" agreement) and the RS outcome recorded in the database ("expected" agreement), we used Cohen's Kappa [36, 37]. The Cohen's Kappa range is between -1 and 1 , whereby 1 indicates a perfect agreement, 0 is what one would expect purely by chance, while negative values indicate a systematic disagreement (i.e., less than that expected by pure chance).

Sensitivity, specificity and Cohen's Kappa statistic were calculated for the results for our NN which consisted of 78 neurons (see above Data Classification Stage and Decision Making), and it was trained for $\leq 100$ epochs. Each training vector (both treatment and retreatment groups) was 30. Every time, the procedure continued until the mean square error was minimized within $\leq 100$ epochs. The detailed presentation of the individual classification tests (before the voting procedure) is cumulatively presented (for 8 individual classification tests) in Table 1.

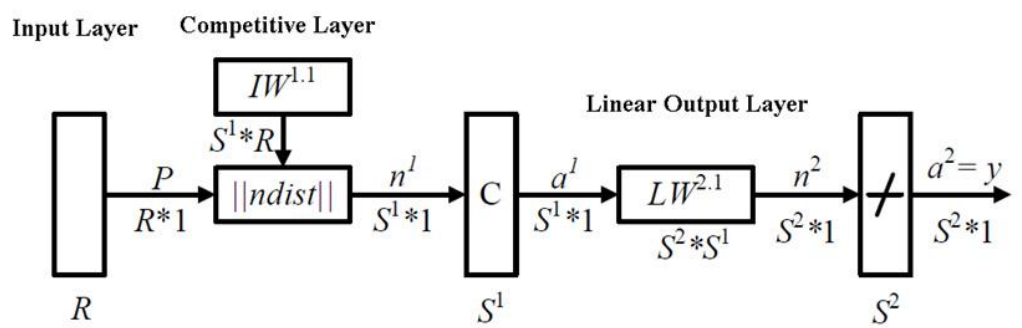

Figure 1. The architecture of the LVQ network. An input vector $P$ with $R$ dimensionality is used. $S^{1}$ is the number of competitive neurons in the layer; $\mathrm{IW}^{\mathbf{1 . 1}}$ is the matrix of the weight coefficients that join the input and competition layer; $\mathbf{n}^{1}$ is the input to the competition layer neuron; $\mathbf{a}^{1}$ symbolizes the competition layer neuron; LW $\mathbf{L W}^{2.1}$ is the matrix of the weight coefficients for the connections which is extracted through the competition between the comparative layers and the linear output layer [38]; $n^{2}$ is the input to the linear output layer; $a^{2}$ is the linear output layer neuron. The output of the training procedure is vector $y$.

Table 1. Cumulative detailed presentation of the individual classification tests

\begin{tabular}{|l|l|l|l|}
\hline Test positive to predict Retreat Cases & $\begin{array}{l}\text { Retreat Case } \\
\text { True Positives (TP) }\end{array}$ & $\begin{array}{l}\text { False Positives (FP) } \\
\mathrm{b}(\mathrm{FP})=4880\end{array}$ \\
\hline Test positive to predict Non-Retreat Cases & $\begin{array}{l}\text { False Negatives (FN) } \\
\mathrm{c}(\mathrm{FN})=144\end{array}$ & $\begin{array}{l}\text { True Negatives (TN) } \\
\mathrm{d}(\mathrm{TN})=13584\end{array}$ \\
\hline & $\begin{array}{l}\text { Total } \\
\mathrm{a}+\mathrm{c}=560\end{array}$ & $\begin{array}{l}\text { Total } \\
\mathrm{b}+\mathrm{d}=18464\end{array}$ & $\begin{array}{l}\text { Total Test Negatives } \\
\mathrm{c}+\mathrm{d}=13728\end{array}$ \\
\hline
\end{tabular}


In table 1, cell "a" contains the cases who were correctly classified as retreat ones, i.e., true positives (TP). Cell " $b$ " contains the cases who were incorrectly classified as retreat ones, i.e., false positives (FP). Cell " $c$ " contains the cases who were incorrectly classified as non-retreat ones, i.e., false negatives (FN). Finally, cell " $d$ " contains the cases who were correctly classified as non-retreat ones, i.e., true negatives (TN). Thus, sensitivity and the specificity were given by:

Sensitivity $=$ TP $/(T P+F N)=416 /(416+144)=0.7429$

Specificity $=\mathrm{TN} /(\mathrm{TN}+\mathrm{FP})=13584 /(13584+4880)=0.7357$

The ranges of the values for the sensitivity and specificity of any individual NN predictions were 0.7429 and 0.7357 respectively.

However, following the voting procedure, which gives the final prediction the values both for the indexes were improved significantly reaching 0.8836 and specificity 0.9186 . With respect to the final prediction, Cohen's Kappa test (0.7658) indicates a substantial agreement between the classification results and the actual outcome $[36,37]$. The software in machine Learning was industrialized by the Laboratory of Information Technologies of the Ionian University in close collaboration with the Ophthalmica Institute of Ophthalmology and Microsurgery, Thessaloniki, Greece (which provided the necessary data for the software development and testing). It can be used as an experimental tool for assessing the probability that corrective treatment may be required following RS for myopia. The software, which can be used either for testing or to explore its capabilities using a researcher's own data is available free on internet, where the relevant disclaimers for using our software are described [39].

\section{DISCUSSION}

Although, major advances in RS took place during the last few decades and an excellent predictability of the refractive outcome $[4,5]$ has been achieved, a small percentage of RS outcomes require corrective retreatment [6-8]. Despite the general decline in the incidence of RS failures, there is still a place for improvement. In addition, the low incidence of retreatments brings about an enhanced anticipation for a perfect RS outcome to patients. Therefore, surgeons must explain to patients that sometimes a corrective RS may be necessary. Otherwise, they open themselves to ethical and legal questions about a potentially unacceptable or inferior (to what is accepted as gold standard), outcome. Our own research efforts have focused on the possible relevance of the numerical representation of five objective patient-related and eight objectives (clinical/operation) procedure-related parameters for identification (by NNs) of myopic patients who are at highrisk for retreatment after RS. In the past, we used the above thirteen parameters to construct a NN which met this task. The NN we have developed predicts potentially unsatisfactory outcomes of RS for myopia with good sensitivity and specificity [12].

Given that the replication of published results is of paramount importance, in the present article we attempted to validate the performance of $\mathrm{NN}$ that we previously developed; For this, we randomly selected 2378 non-retreat and 70 retreat cases from a total of 5210 treatments with a follow-up of over a year and for which we had complete sets of data on record.. All 5210 cases were recorded in the Ophthalmica Institute of Ophthalmology and Microsurgery database of RS (PRK, LASEK, Epi-LASIK or LASIK) and performed between 2010 and 2018.

The five, objective patient-related, parameters that our $\mathrm{NN}$-based algorithm uses are age, axis of astigmatism (in degrees), thickness of the corneal stroma, keratometric values for the horizontal and the vertical axes of the cornea. The other eight objective parameters are related to the conditions under which the operation took place. These can be controlled by the surgeon in his surgical approach to the specific patient. The statistical strength (Cohen's Kappa statistic $=0.7595$ for correct predictions vs actual outcomes) $[36,37]$ and the resulted sensitivity (0.8836) and specificity (0.9186), allow us to be really optimistic about the future of NNs use in the outcome prediction and eventually planning of RS.

\section{CONCLUSION}

We validated our previously published results and confirmed our expectations for the NN we developed. Our results allow us to be optimistic about the future of NNs in predicting the outcome and, eventually, in planning RS.

\section{ETHICAL DECLARATIONS}

- Ethical approval: No need for ethical approval based on nature of study.

- Conflicts of interest: None.

\section{FUNDING}

The author(s) received no specific funding for this work.

\section{ACKNOWLEDGEMENT}

None. 


\section{REFERENCES}

1. Bengio $\mathrm{Y}$, Courville $\mathrm{A}$, Vincent $\mathrm{P}$. Representation learning: a review and new perspectives. IEEE Trans Pattern Anal Mach Intell. 2013;35(8):1798-828. doi: 10.1109/TPAMI.2013.50 pmid: 23787338

2. Schmidhuber J. Deep learning in neural networks: an overview. Neural Netw. 2015;61:85-117. doi: 10.1016/j.neunet.2014.09.003 pmid: 25462637

3. De Fauw J, Ledsam JR, Romera-Paredes B, Nikolov S, Tomasev N, Blackwell S, et al. Clinically applicable deep learning for diagnosis and referral in retinal disease. Nat Med. 2018;24(9):1342-50. doi: 10.1038/s41591-018-0107-6 pmid: 30104768

4. Lee $\mathrm{K}$. Al device for detecting diabetic retinopathy earns swift FDA approval: American Academy of Ophthalmology; 2018 [cited 2018 August 7, 2019]. Available from: https://www.aao.org/headline/first-ai-screen-diabeticretinopathy-approved-by-f.

5. Lin $H$, Long $E$, Ding $X$, Diao $H$, Chen $Z$, Liu R, et al. Prediction of myopia development among Chinese school-aged children using refraction data from electronic medical records: A retrospective, multicentre machine learning study. PLoS Med. 2018;15(11):e1002674. doi: 10.1371/journal.pmed.1002674 pmid: 30399150

6. Achiron A, Gur Z, Aviv U, Hilely A, Mimouni M, Karmona $L$, et al. Predicting Refractive Surgery Outcome: Machine Learning Approach With Big Data. J Refract Surg. 2017;33(9):592-7. doi: 10.3928/1081597X-20170616-03 pmid: 28880333

7. Kohnen T. [Criteria for evaluating and publishing refractive surgery interventions]. Klin Monbl Augenheilkd. 1999;215(6):326-8. pmid: 10637793

8. Koch DD, Kohnen T, Obstbaum SA, Rosen ES. Format for reporting refractive surgical data. J Cataract Refract Surg. 1998;24(3):285-7. doi: 10.1016/s0886-3350(98)80305-2 pmid: 9559453

9. Pokroy R, Mimouni M, Sela T, Munzer G, Kaiserman I. Myopic laser in situ keratomileusis retreatment: Incidence and associations. J Cataract Refract Surg. 2016;42(10):1408-14. doi: 10.1016/j.jcrs.2016.07.032 pmid: 27839594

10. Yuen LH, Chan WK, Koh J, Mehta JS, Tan DT, SingLasik Research G. A 10-year prospective audit of LASIK outcomes for myopia in 37,932 eyes at a single institution in Asia. Ophthalmology. 2010;117(6):1236-44 e1. doi: 10.1016/j.ophtha.2009.10.042 pmid: 20153899

11. Randleman JB, White AJ, Jr., Lynn MJ, Hu MH, Stulting $\mathrm{RD}$. Incidence, outcomes, and risk factors for retreatment after wavefront-optimized ablations with PRK and LASIK. J Refract Surg. 2009;25(3):273-6. doi: 10.3928/1081597X-20090301-06 pmid: 19370822

12. Balidis M, Papadopoulou I, Malandris D, Zachariadis Z, Sakellaris D, Vakalis T, et al. Using neural networks to predict the outcome of refractive surgery for myopia. 4open. 2019;2. doi: 10.1051/fopen/2019024

13. The L. Research matters: challenges of replication. The Lancet. 2017;389(10072). doi: 10.1016/s0140-6736(17)30641-4
14. Mogil JS, Macleod MR. No publication without confirmation. Nature. 2017;542(7642):409-11. doi: 10.1038/542409a pmid: 28230138

15. Goodfellow I, Bengio Y, Courville A. Deep Learning: MIT Press; 2016. 9780262035613

16. Nielsen MA. Chapter 6. Neural Networks and Deep Learning2015.

17. McCaffrey J. Neural Network Training using Particle Swarm Optimization, Dec 18, 2013: Visual Studio Magazine; 2013 [cited 2013 January 7, 2019]. Available from: https://visualstudiomagazine.com/Articles/2013/12/01/NeuralNetwork-Training-Using-Particle-Swarm-

Optimization. aspx? Page $=1 \& p=1$.

18. Courbariaux $M$, Bengio $Y$, David J. Binaryconnect: Training deep neural networks with binary weights during propagations, Advances in Neural Information Processing Systems. 2015:3123-31.

19. Hawickhorst B, Zahorian S, Rajagopal R. A Comparison of Three Neural Network Architectures for Automatic Speech Recognition. Intelligent Engineering Systems Through Artificial Neural Networks. 1995;5:221-6.

20. Kohonen T. Statistical Pattern Recognition Revisited. Advanced Neural Computers1990. p. 137-44.

21. Langari R, Won JS. Intelligent Energy Management Agent for a Parallel Hybrid Vehicle-Part I: System Architecture and Design of the Driving Situation Identification Process. IEEE Transactions on Vehicular Technology. 2005;54(3):925-34. doi: 10.1109/tvt.2005.844685

22. Poulos $M$, Rangoussi $M$, Alexandris $N$, Evangelou $A$. Person identification from the EEG using nonlinear signal classification. Methods Inf Med. 2002;41(1):64-75. pmid: 11933767

23. Ding $\mathrm{CH}$, Dubchak I. Multi-class protein fold recognition using support vector machines and neural networks. Bioinformatics. 2001;17(4):349-58. doi: 10.1093/bioinformatics/17.4.349 pmid: 11301304

24. Kim B-K, Roh J, Dong S-Y, Lee S-Y. Hierarchical committee of deep convolutional neural networks for robust facial expression recognition. Journal on Multimodal User Interfaces. 2016;10(2):173-89. doi: 10.1007/s12193-015-0209-0 25. Hersh PS, Fry KL, Bishop DS. Incidence and associations of retreatment after LASIK. Ophthalmology. 2003;110(4):748-54. doi: 10.1016/s0161-6420(02)01981-4

26. Siedlecki J, Luft N, Kook D, Wertheimer C, Mayer WJ, Bechmann M, et al. Enhancement After Myopic Small Incision Lenticule Extraction (SMILE) Using Surface Ablation. J Refract Surg. 2017;33(8):513-8. doi: 10.3928/1081597X-20170602-01 pmid: 28787515

27. Kruh JN, Garrett KA, Huntington B, Robinson S, Melki SA. Risk Factors for Retreatment Following Myopic LASIK with Femtosecond Laser and Custom Ablation for the Treatment of Myopia. Semin Ophthalmol. 2017;32(3):316-20. doi: 10.3109/08820538.2015.1088552 pmid: 27049689

28. Mimouni M, Vainer I, Shapira Y, Levartovsky S, Sela T, Munzer G, et al. Factors Predicting the Need for Retreatment After Laser Refractive Surgery. Cornea. 2016;35(5):607-12. doi: 10.1097/ICO.0000000000000795 pmid: 26967106 
29. Valdez-Garcia JE, Hernandez-Camarena JC, MartinezMunoz R. 3-Year follow-up after Lasik: assessing the risk factors for retreatment. Int Ophthalmol. 2016;36(1):91-6. doi: 10.1007/s10792-015-0084-4 pmid: 25985886

30. See B, Tan M, Chia SE, Gan WH, Low R, Nah G. Photorefractive keratectomy in young Asian aviators with lowmoderate myopia. Aviat Space Environ Med. 2014;85(1):25-9. doi: 10.3357/asem.3658.2014 pmid: 24479255

31. Gazieva L, Beer MH, Nielsen K, Hjortdal J. A retrospective comparison of efficacy and safety of 680 consecutive lasik treatments for high myopia performed with two generations of flying-spot excimer lasers. Acta Ophthalmol. 2011;89(8):729-33. doi: 10.1111/j.1755-3768.2009.01830.x pmid: 20102346

32. Shojaei A, Mohammad-Rabei H, Eslani M, Elahi B, Noorizadeh F. Long-term evaluation of complications and results of photorefractive keratectomy in myopia: an 8-year follow-up. Cornea. 2009;28(3):304-10. doi: 10.1097/ICO.0b013e3181896767 pmid: 19387232

33. Bragheeth MA, Fares U, Dua HS. Re-treatment after laser in situ keratomileusis for correction of myopia and myopic astigmatism. $\mathrm{Br} J$ Ophthalmol. 2008;92(11):1506-10. doi: 10.1136/bjo.2008.143636 pmid: 18757469

34. Alio JL, Muftuoglu O, Ortiz D, Perez-Santonja JJ, Artola A, Ayala MJ, et al. Ten-year follow-up of laser in situ keratomileusis for high myopia. Am J Ophthalmol. 2008;145(1):55-64. doi: 10.1016/j.ajo.2007.08.035 pmid: 17996210

35. Alio JL, Galal A, Artola A, Ayala MJ, Merayo J. Hyperopic LASIK retreatments with the Technolas laser. J Refract Surg. 2006;22(6):596-603. pmid: 16805124

36. Viera AJ, Garrett JM. Understanding interobserver agreement: the kappa statistic. Fam Med. 2005;37(5):360-3. pmid: 15883903

37. Sim J, Wright CC. The kappa statistic in reliability studies: use, interpretation, and sample size requirements. Phys Ther. 2005;85(3):257-68. pmid: 15733050

38. Papavlasopoulos S, Poulos M, Evangelou A. Feature Extraction from Interictal Epileptic and Non- Epileptic Pathological Eeg Events for Diagnostic Purposes Using Lvq1 Neural Network. Mathematical Methods in Scattering Theory and Biomedical Engineering2006. p. 390-8.

39. Software in Machine Learning by Laboratory of Information Technologies. http://lit.ionio.gr/node/27 date of access: June 1, 2020 